\title{
NATURAL KINDS AND OUR SEMANTIC INTUITIONS ALONG THE ROAD
}

\author{
Thainá Coltro Demartini \\ https://orcid.org/0000-0002-4367-5599 \\ University of Campinas \\ Department of Philosophy \\ Campinas, S.P. \\ Brazil \\ thainacdemartini@gmail.com
}

\begin{abstract}
Article info
CDD: 401

Keywords

Natural kind terms

Scientific properties

Arbitrariness

Determinacy profiles

Context.
\end{abstract}

Received: 13.09.2020; Revised: 23.09.2020; Accepted: 24.09.2020

https://doi.org/10.1590/0100-6045.2020.V43N4.TD

Abstract: This is a comment on Gómez-Torrente's approach to natural kinds and natural kind terms. Here I will focus on his concerns related to the arbitrariness argument and his attempt to formulate a reply to it that maintains most (if not all) of the "Kripke-Putnam orthodoxy" when it comes to the reference-fixing of such terms. Gómez-Torrente concludes that ordinary kind terms have distinct referents from scientific terms. I will challenge one of the premises that he employs in reaching this conclusion: namely, that the difference in determinacy profiles between ordinary natural kinds and scientific kinds is enough to assume that the terms referring to them do not share their referents. I also suggest that some kind of contextual interpretation of natural kind terms might provide a nice explanation of those determinacy variations. 
The main goal of Roads to Reference (Gómez-Torrente 2019 ) is to provide a more detailed and fully developed reference-fixing theory than the one sketched by Kripke, Putnam and Kaplan - the pioneers of the direct reference theory (preceded only by Mill). In pursuit of this goal, Gómez-Torrente analyzes cases of referential indeterminacy that are traditionally employed in arguments against the direct reference theory. He does so in order to find a fitting set of roughly sufficient conditions for reference and for reference failure. Among the specific phrases and terms in the spotlight, there are natural kind terms. In considering these terms, Gómez-Torrente mainly addresses indeterminacy cases generated by the arbitrariness argument. In this review, I will sketch the arbitrariness argument, focusing mostly on Leslie's (2013) approach. Then, I will briefly present Gómez-Torrente's take on it and his response. Next, I will tentatively make an alternative proposal, which consists of a contextual interpretation of natural kinds terms. I will also try to highlight a prominent role of cognition over speakers' intentions when referring to objects, especially when they come in groups, i.e. kinds.

Before we proceed to the analysis of the arguments, I must offer an important disclaimer and establish the common ground assumed by Gómez-Torrente and myself. I do not intend to put in jeopardy the ceteris paribus sufficient clauses for reference-fixing that he suggests. My notes concern the metasemantic and metaphysical consequences implied by his theory, and my main concern is to show how tweaking some details may result in a promising alternative view.

The previously mentioned Kripke-Putnam orthodoxy concerns our intuitions regarding natural kinds and natural kind terms. That is to say, both Kripke and Putnam explore our intuition that merely looking like a sample of a certain kind does not suffice for a thing to belong to that kind - 
there must be something else that is shared, namely, an essence. According to such an intuition, the manifest and macroscopic characteristics are secondary to the microstructural and microscopic ones. Hence, according to the orthodoxy, science would be the one held responsible for discovering hidden underlying features, i.e. essences, that are necessary and sufficient for kind membership. Therefore, it is science that discovers what makes individuals share the relation same substance as and that determines the extension of our natural kind terms. That being said, when someone challenges the orthodoxy by challenging their proposed mechanism of reference-fixing, she can have in mind two distinct questions (one semantic and one metaphysical): Do natural kind terms refer to such scientifically discovered properties $^{1}$ ? And - having a sample of a kind in mind or in front of her - is that sample the same substance as a specific natural kind?

\section{THE ARBITRARINESS ARgUMENT}

The strategy of the arbitrariness argument is to assume the mechanism for reference-fixing postulated by Kripke and Putnam and point to a reductio. According to this argument, the referents of natural kind nouns cannot be identified with the referents expressed in scientific discoveries for arbitrariness reasons. The arbitrariness comes from the fact that, as technology and science evolve and we learn about the microstructure of things, the less obvious it becomes that there is a privileged notion of substance or species instead of multiple theoretical options. But there is

1 Throughout this paper, I will use the terms "kind" and "property" interchangeably.

Manuscrito - Rev. Int. Fil. Campinas, v. 43, n. 4, pp. 199-214, Oct.-Dec. 2020. 
no principled reason for choosing one of the theoretical options over the others to match the ordinary natural kinds (with its set of folk beliefs and the terms that refer to them). All the theoretical options seem to be in the vicinity, and each has slightly different boundaries, none of which are privileged or distinguished by scientists.

To argue for such arbitrariness, Leslie (2013) starts by presenting us specialized information on kinds from the biological and chemical realms, like the use of the concept of species, or the understanding of what it is for water to be $\mathrm{H} 2 \mathrm{O}$. Biologists have no unique way of understanding species. Currently, there are at least two distinct interpretations, one taking into account the delimitations determined by the boundaries of an ecological niche, the other focusing on the boundaries of a reproductive community. Obviously, such boundaries may not perfectly coincide, and, therefore, the meaning of the term species as used in each of these theories will not be exactly coextensive. Despite that, none of these accounts of species is privileged in science. So, Leslie says, it would be arbitrary to pick one of them to be equivalent to our ordinary notion of a species. More to the point, in both scientific approaches, the genetic code of individuals is not enough to make for kind membership. In biology, many phenotypes can come from the same genotype and vice-versa. The fact is that there is no one-to-one correspondence between manifest properties and the genetic code of a kind or individual. The genes are activated (or not) according to environmental exposure and gene combinations. A concrete illustration is the species of cutthroat trout (Salmo clarki) - that has several subspecies. Individuals of such species may have a bigger genetic variation within the species than when they are compared to an individual of another species like the rainbow trout.

On the other hand, we have chemical compounds like H2O. Generally, the compositional formulas provide us with 
no more information than the proportion of elements in a molecule. These formulas provide us with no information about the structure of the molecule. Consequently, it leaves out the variations on the geometric molecular structure itself - the so-called isomers - that make for chemically distinct compounds composed of the same elements and proportions. In the case of $\mathrm{H} 2 \mathrm{O}$, it happens to have a single molecular structure. But still, the substance may be found among dissociated ions of hydrogen and oxygen as it is found in liquid water at normal pressure and temperature. Additionally, at normal pressure and temperature, a sample of water is constituted by 3:1 ratio of orthowater to parawater - those being names describing whether or not the spin of protons in the hydrogen atoms of $\mathrm{H} 2 \mathrm{O}$ molecules are paralleled. These patterns of disassociation of ions and bonding in liquid water (as well as in other substances) happen continuously and dynamically as a consequence of external factors like temperature and pressure. In sum, water seems to be a group of dynamically related complex molecules. Distinct procedures in a laboratory are able to physically separate some of those molecules, like in the case of separating orthowater from parawater, whereas other procedures cannot separate them. Given the variation of lower level properties that water goes through according to the variation of the environment, it seems erroneous to say that water is reducible to $\mathrm{H} 2 \mathrm{O}$ molecules. Then again, Leslie says, it is arbitrary to pick one of those variations of $\mathrm{H} 2 \mathrm{O}$ as water over any other.

Although there is so much more that could be said on the topic of these scientific discoveries, I believe this is enough for Leslie's point to come across: science (at least in its current state) does not have a univocal answer to the question of what it is to be the same substance or species as another thing. Hence, there seems to be no principled reason to pick one of the possible meanings of "species" in biology 
or one of the variations of "substance" in chemistry to be synonymous with our daily uses of those terms. As Leslie puts it, "at best, science delivers a number of candidate same substance relations, many of which rely on the macroscopic and the manifest rather than the microstructural' 2 . While the KripkePutnam orthodoxy highlights the fact that manifest and macroscopic properties are not enough for a sample to belong to a kind, Leslie points to the fact that microstructural properties alone are not enough for a sample to belong to a kind. Up to this date, science has shown no one-to-one correspondence between micro and macro properties and no unique concept of substance or species. That fact shows, according to Leslie, that ordinary natural kinds are not reducible to scientific properties. And, for that same reason, ordinary natural kind terms cannot be reduced to scientific terms.

\section{CONSEQUENCES AND GÓMEZ-TORRENTE'S RESPONSE}

Appealing to scientific facts, Leslie has gathered evidence to show that science does not provide a univocal notion of substance or species and, for the sake of avoiding arbitrariness, an ordinary natural kind cannot be reduced to a scientific property. Contrary to the orthodoxy, Leslie makes a case for science not being a provider of necessary and sufficient conditions to identify an ordinary natural kind. As previously mentioned, going against orthodoxy on natural kinds involves metaphysical as well as semantical issues. On the one hand, Leslie's argument shows that science cannot discover the essence of ordinary objects. It may discover necessary properties, but this is not the same as unveiling their essences, as Putnam and Kripke claim - in

${ }^{2}$ LESLIE, 2013, p.144. 
terms of necessary and sufficient conditions. On the other hand, semantically, if two things are distinct in nature, the extension of the terms that refer to those two things are not coextensive. So, there is no synonymy relation when it comes to ordinary natural kind terms and scientific kinds.

I agree with Gómez-Torrente that proving that ordinary natural kinds are not reducible to scientific kinds does not entail that natural kinds terms do not refer at all. According to the orthodoxy itself, nothing in the reference-fixing criteria requires from the speakers the knowledge of the essence of a kind in order to successfully refer to that kind. All that is required is that, regarding the use of the term, the speaker does not form intentions that, on the whole, conflict with the intentions of the community of users of that term at large. ${ }^{3}$ The direct reference theory allows for significantly meager intentions, as long as they are shared with the community of speakers. Whether it is a singular term or a general term like natural kind terms, a name does not have to be synonymous with any uniquely satisfied description in order to successfully refer. Also, ordinary natural kind terms do seem to work successfully in our language - we are able to communicate using such terms. So, it seems misguided to say they do not refer at all. For that reason, Gómez-Torrente applies a gestalt switch to the arbitrariness argument. He argues that it can be understood "not as eliminativist arguments showing that no reference is fixed because the choice among scientifically precise structural kinds is arbitrary, but as revealing intuitive constraints on the kinds possibly referred to" ${ }^{4}$. The objection is directed to the reduction of one kind to the other, but it does not say

${ }^{3}$ For more details on reference-fixing criteria see GÓMEZTORRENTE, 2019, p.160.

${ }^{4}$ GÓMEZ-TORRENTE, 2019, p.173. 
anything about the referent of ordinary natural kind terms referring to some kind that is not ontologically reducible to scientific kinds.

The existent variation in meaning between ordinary natural kind terms and scientific ones is more easily detected in terms of their determinacy level. The determinacy profile of ordinary natural kind terms shows a higher degree of vagueness than the profiles of scientific kinds terms. That is because science provides us with microstructural descriptions of the kinds they name. Therefore, a speaker knows that when she talks about orthowater, she is referring to molecules of $\mathrm{H} 2 \mathrm{O}$ with a spin of protons in the hydrogen atoms that are not paralleled. Consequently, orthowater is determinately $\mathrm{H} 2 \mathrm{O}$ while it is indeterminately water. If the paradigmatic case of water that first stablished the reference of the term "water" is constituted by 3:1 ratio of orthowater to parawater, then another sample with the same ratio may be considered determinately water (although it's not determinately reducible to that precise structure). And the same would happen in the biological realm, as in the case of tigers. An animal with genetic code $\mathrm{T}$ determinately has the genetic code $\mathrm{T}$ and is indeterminately a tiger, unless it is part of the paradigmatic case of tiger or has the exact same genetic code of the paradigmatic sample.

Is the distinction in determinacy profiles enough to state metaphysically distinct kinds - one vague, the other one sharp? Gómez-Torrente's answer to that question is yes. According to him, ordinary natural kinds metaphysically exist as sui generis kinds. In opposition to Gómez-Torrente's view, I take the fact that scientific properties can still be presented as necessary properties of ordinary natural kinds as a hint that the answer to such a question is no. The fact that they share some necessary properties keeps their modal profiles closely connected, and I take that as evidence of 
ordinary natural kind terms and scientific kind terms sharing their referents.

\section{Alternative Route AND ITS Motivations}

The assumption of an "all the way down" description of the structure of things and kinds (of particulars being "ultimate" or "basic") is something that needs to be argued for - and requires a lot of metaphysical theorizing. I do not think that the assumption that science will eventually find the ultimate minimal constituents of objects is part of the Kripke-Putnam orthodoxy. Kripke himself says that "although we can try to describe the world in terms of molecules, there is no impropriety in describing it in grosser entities" 5 . Hence, he attests that, unless there is a basic particular, no description should be regarded as privileged. He exemplifies his view on the issue by mentioning facts about nations. Perhaps a fact about Germany during the War cannot be reduced to any statement about individual Germans but in some sense facts about a nation are not "over and above" facts about people. ${ }^{6}$

\footnotetext{
${ }^{5}$ KRIPKE, 1980, p.51.

${ }^{6}$ My guess is that people take this assumption as part of the Kripke-Putnam orthodoxy because of a misconception of an idea proposed in Salmon (2005). In this classic work, Salmon argues that if Kripke and Putnam did not concede to an a priori justification of scientific properties being necessary to natural kinds, they would have to claim the sufficiency of these same scientific properties to identifying natural kinds. That would be the only way to attest to the necessity of such properties without presupposing it - as a hidden metaphysical premise of their theory. Nevertheless, assuming that nowadays most theorists would agree that essentialist metaphysics is not entailed by the semantic analysis
} 
Not considering this assumption to be among the cornerstones of the Kripke-Putnam orthodoxy allows us to outline an alternative view in which ordinary natural kind terms and scientific kind terms refer to the same metaphysical kinds, on one level or another. Contextual considerations enable us to understand both scientific and ordinary terms as referring to the same metaphysical kind. Semantically, we can have contextual considerations openly expressed by acknowledging that natural kind terms include an indexical component in their meanings. Metaphysically, we can argue that facts about water may not be reducible to facts about $\mathrm{H} 2 \mathrm{O}$ but in some sense water facts are not facts "over and above" facts about its constituent molecules. Additionally, there is no guarantee that science will not discover even lower level distinctions and finer grained properties than the ones currently known. For instance, with the advance of technology, scientists might discover that there are two kinds of orthowater. Context sensitivity will help us to incorporate that new discovery in the spectrum of reference without changing the meaning of the previously stated kind terms as "water", "H2O" and "orthowater" none of them needs to be considered as referring to "basiclevel" kinds.

As Gómez-Torrente says, "the possibility of different contextual standards is compatible with the objectivity of the properties characterized and constituted by such standards" . If we assume that natural kinds are properties that involve specific conditions of observation and measurement at each time they are referred to by a term, and that those specific conditions are objectively specifiable, then

but instead presupposed by it, there is no room for sufficiency requirements in the orthodoxy.

7 GÓMEZ-TORRENTE, 2019, p.197 
they can also be considered objective properties themselves. Even properties popularly considered objective (such as extension) can be interpreted as involving conditions of observation. For instance, regarding its length or shape, the coastline of Britain is always determined (and varies) according to the level of resolution. It is intuitive that its shape will seem different when seen from an airplane, as opposed to when walking along it on foot. The amount of detail will increase, as will its length if we measure it with a ruler and consider every little corner, instead of measuring it from the airplane by using a scaling factor of the perception.

Gómez-Torrente is aware of those perceptual factors which do influence even the most straightforwardly objective properties. So, instead of postulating two distinct ontological kinds, the ordinary and the scientific, why not consider a view that includes a context-sensitive semantics of natural kind terms, while also having fewer ontological commitments? The main motivation for my tentative approach is to favor simplicity, and thus avoid commitment to an objective distinction between ordinary natural kinds and biochemically, chemically or physically precise structural scientific kinds. Also, I believe that the vagueness of the speaker's intentions when referring to ordinary natural kinds can be explained by the similarly vague contextual standards. But, more importantly, ordinary kind standards can be linguistically explained in association with human cognition and our default mode of generalization.

\section{NATURAL KIND TERMS AND GENERALIZATIONS}

Even if we do not have a metaphysically principled reason for considering ordinary natural kinds as basic level categories, evidence coming from human psychology favors the thesis that there may be a cognitive reason for treating 
them as such. To talk about kinds, a speaker is constantly making generalizations from the known particulars to all things belonging to a kind. Also, the idea of a kind itself requires knowing at least two elements and how they combine to define the new kind. In fact, the ability to generalize is prior to the acquisition of language. Infants as young as twelve months are already capable of category-wide generalizations on the basis of experience with a few instances of the category (Graham, Kilbreath, and Welder 2001).

Leslie (2007) has argued that the default generalization mechanism shows up in language use in the form of generics. Examples of generics are sentences such as "Tigers are striped", "Ducks lay eggs", and "Mosquitoes carry the West Nile Virus". Even though the truth conditions of these sentences are complex and hard to grasp, we do have straightforward intuitions about whether they are true or false. Empirical studies appear to confirm generics as primitive components of our language (Gelman 2003; Roeper, Strauss, and Pearson 2006). It is a fact that young children learn and master generics before they do quantificational sentences. Also, Chomsky (2000) has suggested that unmarked surface forms on semantic formulations do invoke cognitive default mechanisms. And it is not unique to English that generics do not present a marked modifier in sentences like the quantifiers "all", "some", "at least one" do. Therefore, the data can be interpreted as favoring Leslie's claim that the default generalization mechanism is translated in language use by generics.

Assuming this position is correct, by investigating the semantics of generics, we can attain a deeper understanding about our default generalization mechanism. The truth of a generic sentence such as "Tigers are striped" seems to require no more than that the majority of the members of 
this kind possesses the relevant property. But not all generics can be understood as statements of "all normal cases" in a kind. This does not work, for example, for other true generic sentences such as "Ducks lay eggs". So, we have to include a special requirement to deal with characteristic dimensions of a kind, such as their characteristic methods of reproduction, or diet or the like. The characteristic dimensions are not considered just like any other neutral characteristic of a kind - in the case above the generic is true even if only half of the population has it (like only female ducks lay eggs). Still, sentences like "Mosquitoes carry the West Nile Virus" do not seem to fit either of the previous requirements. To address cases like this it is necessary to include a clause for particularly striking or appalling information. If the characteristic under consideration can potentially intervene in human life and lifestyle, then the generic sentence that mentions it will also be true.

Similarly, we can anticipate that the human default generalization mechanism is not solely concerned with how many ${ }^{8}$. It is actually intuitive to think that the efficiency of a default mechanism of generalization would consider more than the regularity of characteristics shown by a kind. For instance, for survival purposes, it would be better to also include particularly striking or appalling information to generalized contents. The default considerations of characteristic dimensions and appalling information help to understand why ordinary natural kinds are taken as "basiclevel" kinds of the mechanism. The characteristic dimensions detected by our minds are part of an information gathering process by means of which we identify

\footnotetext{
8 "That our intuitions about the truth of a generic can be altered by such facts that do not pertain to cardinalities reflects the fact that generics depend on far more than cardinalities for their truth and falsity." (LESLIE, 2007, p.393)
} 
dimensions of regularities across kinds, such as identifying the fact that all animal kinds have their own method of reproduction, produce peculiar noises, etc. And by doing so we focus on eye catching distinctions across those animal kinds and strengthen our sense of a shared commonality within each of them. On the other hand, the appalling information gathered by the mechanism contributes to restricting the generalizations. For instance, "Mosquitoes carry the West Nile Virus" is intuitively true but "Animals carry the West Nile Virus" is not. Therefore, the particularly striking characteristic would focus on the smallest groups that are disposed to satisfy the characteristics and end up selecting those "basic-level" kinds that we know as ordinary natural kinds.

I suggest that this default mechanism and its converging requirements are responsible for our grasp on ordinary natural kinds. Our default intuitive practices use them as basic-level kinds in order to make generic predictions. There is research that attests to the fact that even preschool children have a grasp on natural kinds and prefer kind membership rather than perceptual similarity in making inferences (Gelman and Coley, 1990). It seems plausible that speakers' intentions when referring with a natural kind term would be directed and restricted by this primitive cognitive mechanism. And that could justify the vagueness of these intentions, since they are based on a cognitive process that does not necessarily privileges characteristics that the majority of the kind possess. Instead, it involves characteristic dimensions that are selected within human epistemological constraints (characteristics that can be detected by our perception without any tools, such as microscopes). Hence, given our vague contextual standards, it makes sense of our initial intentions. Those intentions can be meager, as intended by Kripke. But they can also grow 
together with the growth of our standards once we get in touch with scientific advances.

\section{CONCLUSION}

Of course, the expansion of empirical research concerning language acquisition and the mind's default generalization mechanism have a lot to contribute on the topic. Nevertheless, given the empirical facts at our disposal, this was a tentative suggestion to shrink our ontology (and, therefore, our ontological problems) without interfering with Gómez-Torrente's innovative semantic proposals. Whether it is a successful alternative or not, it is at least a proof that Gómez-Torrente's book - beyond its valuable contributions for the reference-fixing theory to friends of the direct reference tradition - is capable of starting discussions beyond the realm of semantics.?

\section{REFERENCES}

CHOMSKY, N. (2000), New Horizons in the Study of Language and Mind. Cambridge: Cambridge University Press.

GELMAN, S.A. (2003), The Essential Child. New York: Oxford University Press.

9 I am grateful to my colleagues from Unicamp and those participating in this edition of Manuscrito for fruitful discussions, specially to Mario Goméz-Torrente. I am also grateful to David Mokriski, Hugo Vedovato, Joshua Thorpe, Marco Ruffino and Matheus Valente for their careful reading and comments on earlier drafts. 
. \& COLEY, J.D. (1990) "The importance of knowing a dodo is a bird: Categories and inferences in 2-year-old children". Developmental psychology 26, pp. 796-804.

GÓMEZ-TORRENTE, M. (2019), Roads to Reference. An Essay on Reference Fixing in Natural Language. Oxford, Reino Unido: Oxford University Press.

GRAHAM, S.A., KILBREATH, C.S. \& WELDER, A.N. (2001), "Words and Shape Similarity Guide 13month-olds' Inferences about Nonobvious Object Properties". In J.D. Moore and K. Stenning (eds.) Proceedings of the Twenty Third Annual Conference of the Cognitive Science Society. Erlbaum. Hillsdale. pp. 352357.

KRIPKE, S. (1980), Naming and Necessity. Cambridge, Mass.: Harvard University Press.

LESLIE, S.J. (2007), "Generics and the Structure of Mind". Philosophical Perspectives 21, pp.375-403, 2007. . (2013), "Essence and Natural Kinds: When Science Meets Preschooler Intuition". Oxford Studies in Epistemology 4, pp. 108-166.

ROEPER, T. STRAUSS, U. \& PEARSON, B.Z. (2006), "The acquisition path of the determiner quantifier every: Two kinds of spreading". In Current Issues in First Language Acquisition, University of Massachusetts Occasional Papers 34, pp. 97-129.

SALMON, N.U. (2005), Reference \& Essence. New York: Prometheus Books, 2005.

$(\infty)$ EY 